\title{
Corrections
}

\section{Correction: Simmons et al., A Small Molecule TrkB Ligand Reduces Motor Impairment and Neuropathology in R6/2 and BACHD Mouse Models of Huntington's Disease}

In the article "A Small Molecule TrkB Ligand Reduces Motor Impairment and Neuropathology in R6/2 and BACHD Mouse Models of Huntington's Disease” by Danielle A. Simmons, Nadia P. Belichenko, Tao Yang, Christina Condon, Marie Monbureau, Mehrdad Shamloo, Deqiang Jing, Stephen M. Massa, and Frank M. Longo, which appeared on pages 18712-18727 of the November 27, 2013 issue, one of the authors failed to disclose a financial conflict of interest. Dr. Stephen Massa was listed as an inventor on a patent (US7723328 B2) covering the small molecules described in the paper. The reviewers and the editors were unaware of this interest at the time the paper was reviewed.

DOI: $10.1523 / J N E U R O S C I .0053-14.2014$

\section{Correction: Tep et al., Oral Administration of a Small Molecule Targeted to Block proNGF Binding to p75 Promotes Myelin Sparing and Functional Recovery after Spinal Cord Injury}

In the article "Oral Administration of a Small Molecule Targeted to Block proNGF Binding to p75 Promotes Myelin Sparing and Functional Recovery after Spinal Cord Injury” by Chhavy Tep, Tae Hee Lim, Pyung On Ko, Sami Getahun, Jae Cheon Ryu, Virginia M. Goettl, Stephen M. Massa, Michele Basso, Frank M. Longo, and Sung Ok Yoon, which appeared on pages 397-410 of the January 9, 2013 issue, one of the authors failed to disclose a financial conflict of interest. Dr. Stephen Massa was listed as an inventor on a patent (US7723328 B2) covering the small molecules described in the paper. The reviewers and the editors were unaware of this interest at the time the paper was reviewed.

DOI: 10.1523/JNEUROSCI.0054-14.2014

\section{Correction: Schmid et al., A TrkB Small Molecule Partial Agonist Rescues TrkB Phosphorylation Deficits and Improves Respiratory Function in a Mouse Model of Rett Syndrome}

In the article "A TrkB Small Molecule Partial Agonist Rescues TrkB Phosphorylation Deficits and Improves Respiratory Function in a Mouse Model of Rett Syndrome” by Danielle A. Schmid, Tao Yang, Michael Ogier, Ian Adams, Yatin Mirakhur, Qlfang Wang, Stephen M. Massa, Frank M. Longo, and David M. Katz, which appeared on pages 1803-1810 of the February 1, 2012 issue, one of the authors failed to disclose a financial conflict of interest. Dr. Stephen Massa was listed as an inventor on a patent (US7723328 B2) covering the small molecules described in the paper. The reviewers and the editors were unaware of this interest at the time the paper was reviewed.

DOI: 10.1523/JNEUROSCI.0055-14.2014

\section{Correction: Massa et al., Small, Nonpeptide p75NTR Ligands Induce Survival Signaling and Inhibit proNGF-Induced Death}

In the article "Small, Nonpeptide p75NTR Ligands Induce Survival Signaling and Inhibit proNGF-Induced Death" by Stephen M. Massa, Youmei Xie, Tao Yang, Anthony W. Harrington, Mi Lyang Kim, Sung Ok Yoon, Rosemary Kraemer, Laura A. Moore, Barbara L. Hempstead, and Frank M. Longo, which appeared on pages 5288-5300 of the May 17, 2006 issue, two of the authors failed to disclose a financial conflict of interest. Dr. Stephen M. Massa and Dr. Frank M. Longo were inventors associated with a US provisional patent application $(60 / 688,767)$ covering the small molecules described in the paper. The reviewers and the editors were unaware of this interest at the time the paper was reviewed.

DOI: 10.1523/JNEUROSCI.0056-14.2014 